\title{
Enseignement de la médecine de premier recours - décision obligatoire de la CIMS du 24 mars 2004
}

C. Bader, Président de la CIMS

\section{Historique}

Lors de la séance de la CIMS (Commission interfacultés médicale suisse) du 13 mars 2002, le Dr B. Brinkley (Genève) et le Dr D. Ackermann (Zürich), représentants à la CIMS de la médecine générale, présentent leur proposition de créer un groupe de travail de la CIMS visant à harmoniser les conditions cadres de l'enseignement de la médecine générale dans les Facultés de médecine suisses. Ces conditions sont en effet extrêmement variables.

Après discussion, le Prof. Peter Suter, alors président de la CIMS, a conclu que la proposition était pertinente et recevable. L'exercice-pilote d'accréditation avait en effet démontré que l'enseignement de la médecine ambulatoire était sous-développé dans toutes les Facultés. Il propose donc:

- de mieux définir ce qui caractérise le domaine «médecine générale / médecine de premier recours / médecine de famille»;

- de procéder à un état des lieux de l'enseignement actuel dans les Facultés;

- de proposer des mesures permettant d'améliorer la situation.

Un groupe de travail «Primary care» est constitué comme suit: Prof. Dr. W. Vetter, Studiendekan Klinik Zürich, Président; Prof. H. J. Kaiser, Studiendekanin, Basel; Prof. V. Im Hof, Vizedekan Lehre, Bern; Prof. C. Bader, Vice-doyen responsable de l'enseignement, Genève; Prof. F. T. Bosman, Vice-doyen responsable de l'enseignement, Lausanne; Dr. med. P Tschudi, FIAH Basel; Dr. med. A. Rothenbühler, FIAH Bern; Dr B. Brinkley, UEMG Genève; Dr T. Bishof, UMG Lausanne; Dr. med. D. Ackermann, FIHAM Zürich.

Des rapports intermédiaires ont été présentés aux séances de la CIMS du 5 juin 2002 et du 6 novembre 2002. Le 27 mars 2003, un premier document consensus est présenté. Ce document fait l'objet de modifications et est accepté comme décision simple lors de la séance de la CIMS du 11 juin 2003.

\section{Décision simple}

Selon les statuts de la CIMS (V.1), les décisions simples représente une libre appréciation ou recommandation de la CIMS destinée aux Facultés de médecine et institutions affiliées à la CIMS ou adressée à d'autres milieux.

Suite à cette décision, les Facultés ont été consultées. Toutes les Facultés se sont prononcées positivement lors de la séance de la CIMS du 24 mars 2004. Les délégués des Facultés étant tous doyens et vice-doyens responsables de l'enseignement de leurs Facultés respectives, le Président Charles Bader a considéré que la décision obligatoire du 24 mars 2004 concernant la médecine de premier recours devenait de facto une décision obligatoire.

\section{Décision obligatoire}

Selon les statuts de la CIMS (V.2), à la demande de tous les délégués d'une Faculté ou du président de commission, une décision simple prise sur un objet figurant à l'ordre du jour peut, dans une seconde votation, être déclarée obligatoire pour toutes les Facultés affiliées. Seuls les délégués des Facultés ont le droit de vote pour déclarer une décision obligatoire. Chaque délégué a droit à une voix. La majorité des délégués présents fait foi. Le président n'a pas le droit de vote mais il départage les voix en cas d'égalité.

Lorsqu'une décision obligatoire a été votée, sa teneur est communiquée par écrit aux Facultés et un délai leur est imparti pour la ratifier par écrit. A défaut d'un tel accord écrit notifié par une Faculté dans le délai prescrit, son assentiment est admis. Lorsque la majorité des Facultés est acquise, la décision obligatoire est adoptée et mise en vigueur lors de la prochaine séance de la Commission.

Si dans les délais voulus une décision obligatoire n'est pas confirmée expressément ou tacitement par la majorité des Facultés, elle est nulle et non avenue. 
On trouvera ci-dessous le document final.

\section{Rapport du groupe de travail de la CIMS «médecine de premier recours durant les études de médecine»}

\section{Définition}

Conformément au document de consensus de la WONCA (2002), la médecine générale / médecine de famille (ci-après médecine de premier recours) constitue une discipline académique et scientifique qui comprend ses propres contenus de formation pré-graduée, post-graduée et continue, ses propres domaines de recherche et activités cliniques, ainsi que des principes méthodologiques basés sur la preuve. En outre, c'est une discipline clinique orientée vers les soins de base. Sont indiqués ci-dessous les éléments de la liste générale des caractéristiques de la médecine de premier recours du document de la WONCA pour lesquels une spécificité d'enseignement est mentionnée:

- évaluation en première ligne de problèmes de santé non préalablement sélectionnés;

- fonction de triage;

- prise en charge de patients dans le réseau social / visites à domicile;

- suivi à long terme et continuité;

- accompagnement ambulatoire aux patients en fin de vie;

- contacts étroits avec les organismes de santé publiques/ les écoles.

\section{Contenu de l'enseignement}

En principe, chaque université définit son propre curriculum. C'est ainsi que le curriculum peuvent varier d'une université à l'autre. L'intégration des contenus de la médecine de premier recours devrait si possible se faire dans toutes les années d'études tout en respectant les conditions spécifiques à chaque université.

A cet effet le groupe de travail estime pertinent que, dans chaque faculté, des collègues qualifiés et coopératifs participent à tous les groupes de travail et commissions directement concernés par l'enseignement, après approbation par les instances facultaires concernées.

Lors de la répartition par discipline des objectifs d'apprentissage du catalogue suisse des objectifs, on devrait prendre en compte, en plus des principes susmentionnés plus haut, la définition de la médecine de premier recours et celle des caractéristiques spécifiques pour l'enseignement. En principe, les objectifs d'apprentissage marqués du label «G» dans le catalogue des objectifs peuvent être enseignés par des méde- cins de premier recours. La réglementation précise en la matière est du ressort de chaque faculté.

\section{Structure recommandée}

Le groupe de travail recommande d'instaurer, au niveau universitaire, une unité qui prendrait en charge, dans la continuité, les tâches et la responsabilité du domaine de l'enseignement et de la recherche en médecine de premier recours.

4. Conditions cadre des activités d'enseignement (décision des vice-doyens responsable de l'enseignement médical en Suisse)

Le terme «chargé d'enseignement de la Faculté de médecine de l'université» ou un titre analogue sera proposé comme titre officiel des universités pour le domaine de la médecine de premier recours, en accord avec les instances facultaires correspondantes.

Une rémunération appropriée des activités d'enseignement est prévue dans le cadre de la charge d'enseignement, selon la situation propre à chaque Université.

Version du 11 juin 2003 après la réunion avec la CIMS.

\section{Informations de base sur la CIMS}

\section{Commission interfacultés médicale suisse (CIMS)}

Créée en 1964, la Commission interfacultés médicale suisse (CIMS) est un groupe de travail des facultés responsables des études et de la formation du médecin, de même que d'autres organisations participant ou s'intéressant à ces tâches.

\section{Composition}

Elle est constituée de 36 délégués des Facultés, d'Associations (FMH, AMAC, AEMS), d'Offices (OFSP, OFASA) et autres institutions (Comité directeur des examens fédéraux pour les professions médicales, CUS). Elle peut faire en outre appel à des experts et s'adjoindre des invités.

\section{Tâches}

La Commission interfacultés médicale suisse a pour tâche:

1. D'assurer l'information réciproque des facultés et des organisations affiliées à la Commission interfacultés, afin

2. de coordonner les programmes et les méthodes d'enseignement des diverses facultés en vue de la formation requise pour le diplôme fédéral de médecine au sens de la 
loi fédérale concernant l'exercice des professions de médecin, de pharmacien et de vétérinaire dans la Confédération suisse, du 19 décembre 1877/21 décembre 1886;

3. de traiter les questions se rapportant au but des études médicales, à la formation des étudiants en médecine, aux examens pour le diplôme fédéral de médecine et à la formation postgraduée des médecins;

4. de représenter les facultés et les organisations affiliées, dans leur ensemble, auprès des autorités fédérales et cantonales, notamment auprès de la Conférence universitaire suisse, du Conseil suisse de la science et du Comité directeur des examens fédéraux pour les professions médicales.

\section{Modalités de travail}

1. La Commission interfacultés se réunit au moins deux fois par an en séance ordinaire.

3. La Commission interfacultés atteint son quorum lorsque la majorité des facultés de médecine sont intégralement représentées.

8. Financement

Les facultés affiliées contribuent aux frais généraux habituels (travail, ports et matériel de secrétariat, dépenses du président, etc.). La commission fixe le montant de ces contributions en tenant compte de la participation des diverses facultés à l'ensemble de l'enseignement. Les comptes annuels sont soumis pour approbation à la commission.

Les facultés, associations, offices et institutions rémunèrent selon leur libre appréciation le travail et les frais de leurs délégués.

Pour couvrir des débours extraordinaires entrant dans le cadre du programme des travaux établi par la commission, notamment: documentation, recours à des experts du dehors et autres mesures, des contributions spéciales peuvent être perçues. Elles devront cependant être fixées au moment même où la commission décide de ces mesures et ne seront pas exigibles après coup.

V.3 Application d'une décision obligatoire

Les facultés affiliées à la commission interfacultés sont tenues d'appliquer dans les délais utiles une décision obligatoire et d'y adapter au besoin leurs règlements internes. Sont réservés néanmoins les lois cantonales et les décrets supérieurs de l'université engageant la faculté.

Lorsqu'en raison de dispositions contraires découlant d'une loi ou d'un décret supérieur de son université une faculté n'est pas en mesure d'appliquer une décision obligatoire, elle en est dispensée si elle apporte la preuve d'être intervenue avec la fermeté voulue auprès des autorités compétentes en leur proposant d'adopter la modification en cause.

\section{Répartition des tâches avec le Comité directeur et la Conférence universitaire suisse}

\section{Comité directeur}

Selon la loi concernant l'exercice des professions médicales et l'ordonnance générale concernant les examens fédéraux des professions médicales, le Comité directeur veille à ce que les examens fédéraux des professions médicales se déroulent conformément aux prescriptions et à ce que les candidats atteignent les buts assignés à la formation (exigences scientifiques).

\section{Conférence universitaire suisse}

Elle traite pour le domaine de la médecine les problèmes ci-après:

- information des bacheliers;

- capacités d'accueil;

- accès aux études de médecine;

- organisation de la campagne de préinscription.

La CIMS et le Comité directeur sont les deux organismes où sont discutées toutes modifications de l'enseignement ou des examens dans le cadre des études de médecine et de médecine dentaire. Toutes les réformes introduites dans les études de médecine en Suisse l'ont été avec l'aval de ces deux organismes dont il n'est pas exagéré de dire qu'ils ont été également des moteurs initiaux de ces réformes. 